\title{
DIAGNOSTICO DE OSTEOPOROSIS POR MEDIO DE DENSITOMETRIA OSEA Y VALORACION DE RIESGO DE FRACTURA
}

\section{Osteoporosis diagnosis through and valuation bone densitometry fracture risk}

*Georgina González Pinto, ** Manuel González Pieri, **Martha Rodríguez.

\section{RESUMEN}

La Osteoporosis es una enfermedad generalizada del sistema esquelético caracterizado por la pérdida de masa ósea y por el deterioro de la microarquitectura del tejido óseo, que compromete la resistencia ósea y que condiciona como consecuencia, mayor fragilidad ósea y susceptibilidad a fracturas. La medición de densidad ósea puede ser usada para establecer o confirmar el diagnóstico de osteoporosis y predecir el riesgo futuro de fracturas. A menor densidad mineral ósea mayor riesgo de fractura. Objetivo: Identificar la densidad ósea y riesgo de fracturas en pacientes que acudieron a Mediscan para la realización de Densitometría Ósea. Pacientes y métodos: Estudio descriptivo, retrospectivo, realizado en 2317 pacientes que acudieron a Mediscan en el periodo de julio 2012 a enero 2016 a estudio de densitometría ósea. Resultados: de los 2317 pacientes, 2205 (95\%) fueron mujeres y $112(5 \%)$ hombres. De las mujeres mayores de 50 años; $11.82 \%$ presentó osteopenia y $44.74 \%$ osteoporosis y de los hombres el $0.51 \%$ presentó osteopenia y $1.97 \%$ osteoporosis. Conclusión: la correcta identificación de la pérdida de masa ósea a través de factores de riesgo de nuestra población y segmentarla nos ayuda desde el punto de vista médico a ofrecer un mejor diagnóstico y adecuada orientación a nuestros pacientes.

\section{PALABRAS CLAVE}

Densidad ósea, Osteoporosis, factores de riesgo.

*Medico Radióloga, Mediscan

${ }^{* *}$ Medico General, Mediscan

Dirigir correspondencia a: mjpieri9@gmail.com

Recibido: 12 de mayo 2016, Aprobado: 08 de diciembre 2016

\section{ABSTRACT}

Osteoporosis is a generalized disease of the skeletal system characterized by the loss of bone mass and by the deterioration of the microarchitecture of bone tissue, which compromises bone resistance and which results in increased bone fragility and susceptibility to fractures. Bone density measurement can be used to establish or confirm the diagnosis of osteoporosis and to predict the future risk of fractures. The lower the bone mineral density the higher the risk of fracture. Objective: To identify the bone density and risk of fractures in patients who came to Mediscan for Bone Densitometry. Patients and Methods: This was a descriptive, retrospective study of 2317 patients who visited Mediscan in the period from july 2012 to january 2016 in the study of bone densitometry. Results: Of the 2317 patients, 2205 (95\%) were women and 112 (5\%) men, in the population older than 50 years; $11.82 \%$ had osteopenia and $44.74 \%$ osteoporosis. In men $0.51 \%$ presented osteopenia and $1.97 \%$ presented osteoporosis in men. Conclusion: The correct identification of the loss of bone mass through risk factors of our population and segmenting it helps us from the medical point of view to offer a better diagnosis and adequate orientation to our patients.

\section{KEYWORDS}

Bone Density, Osteoporosis, risk factors.

\section{INTRODUCCIÓN}

La Osteoporosis (OP) se define como una enfermedad generalizada del sistema esquelético caracterizado por la pérdida de masa ósea y deterioro de la microarquitectura del tejido óseo, que compromete la resistencia 
ósea y condiciona mayor fragilidad ósea y susceptibilidad a fracturas, ${ }^{(1)}$ esta definición es propuesta por el National Institute of Health (NIH) que actualiza el concepto establecido por consenso en 1993 en Hong-Kong. La definición de la OP de los Institutos Nacionales de Salud (NIH) integra 2 características: la cantidad de masa ósea (componente cuantitativo) y el concepto de resistencia-fragilidad ósea (componente cualitativo) ${ }^{(2,3)}$

En 1989, un comité científico para la fundación de la osteoporosis, describió 4 situaciones clínicas, en las cuales el conocimiento de la masa ósea y el riesgo de fractura deben ser tomados en cuenta para las decisiones de manejo clínico. Estos son:

a) Deficiencia de estrógeno.

b) Sospecha de osteopenia en radiografía convencional o anormalidades vertebrales.

c) Hiperparatiroidismo primario asintomático.

d) Terapia a largo plazo con corticoesteroides. ${ }^{(3)}$

El máximo nivel de masa ósea se alcanza entre los 25-30 años y está determinado por múltiples factores, siendo el principal, el factor genético. También son importantes los factores nutricionales, la actividad física, factores endocrinos y género femenino. Este equilibrio normal entre la síntesis y la reabsorción ósea mantiene constante la masa ósea. A partir de los 40 años se observa una lenta reducción de la densidad de la masa ósea en ambos sexos (aproximadamente 0.3-0.5\% al año). La densidad de masa ósea (DMO) se expresa en gramos de mineral por unidad de superficie (cm2). La DMO puede estimarse mediante varias técnicas: radiología convencional, ultrasonido de calcáneo, tomografía cuantitativa y densitometría ósea; este último es el método que debe de considerarse como estándar, debido a que además de medir la densidad mineral ósea de la columna lumbar y del cuello femoral, cuantifica el riesgo de fractura, una vez establecido un tratamiento, su control permite la valoración de ganancia ósea. ${ }^{(4)}$
El hueso es un tejido que está en constante formación y destrucción a lo largo de toda la vida. Este fenómeno se conoce como remodelado óseo y se lleva a cabo por medio de la unidad de remodelación ósea que consiste en un conjunto de células: osteoclastos y osteoblastos. El remodelado óseo tiene dos funciones: en primer lugar, sustituir el tejido óseo viejo por nuevo, aumentar la resistencia del esqueleto a las fracturas; y en segundo lugar asegura la disponibilidad de minerales como calcio, fosforo o magnesio para ser transportados del hueso al líquido extracelular y viceversa, de acuerdo con las necesidades del organismo. Las células que participan en el remodelado óseo son de diversos tipos, pero dos de ellas son las protagonistas principales del proceso: los osteoclastos, que son macrófagos especializados en destruir hueso, fenómeno denominado "resorción ósea" y los osteoblastos, células derivadas del tejido conectivo que se encargan de formar hueso. ${ }^{(5)}$

En la osteoporosis se produce una disfunción de las unidades de remodelado óseo que a su vez se debe fundamentalmente a dos tipos de alteraciones; la primera consiste en el establecimiento de un "balance negativo" y la segunda en un aumento del número de unidades de remodelación, que da lugar a lo que se designa como "aumento de recambio óseo". (5)

Los factores de riesgo para Osteoporosis son: edad, menopausia, genética, baja masa corporal (IMC <18), estilo de vida, baja ingesta de calcio y vitamina $D$, consumo de tabaco y alcohol, baja actividad física, uso de corticoesteroides. ${ }^{(5,6)}$

DENSITOMETRIA OSEA (DMO): La medición de densidad ósea puede ser usada para establecer o confirmar el diagnóstico de osteoporosis y predecir el riesgo de fractura. A menor densidad mineral ósea mayor riesgo de fractura. La medición de densidad mineral ósea puede ser efectuada en cualquier sitio, los sitios elegidos para estas mediciones son: la columna lumbar y cuello femoral. ${ }^{(6)}$ 
La DMO es un método diagnostico basado en una fuente de radiación con un haz de Rayos $X$ focalizado, que estudia la columna vertebral y pelvis, y que representa una metodología numérica, que analiza $\mathrm{g} / \mathrm{cm} 3$ de hueso. No se debe de considerar un estudio anatómico que sustituye a otras técnicas diagnósticas como rayos $\mathrm{X}$ convencional, tomografía cuantitativa dirigida a estudio anatómico de algún segmento en particular ni resonancia magnética nuclear. Facilita el diagnóstico de osteoporosis por ser una metodología rápida y no invasiva, y es el único método aceptado como seguimiento en pacientes con esta enfermedad para comparación de su recuperación ósea en relación con los porcentajes de recuperación de densidad mineral ósea. ${ }^{(6,7)}$ Respecto a su capacidad para predecir fracturas, principal complicación de la osteoporosis, presenta una elevada especificidad pero una escasa sensibilidad (no es útil como prueba de cribado) y una baja utilidad predictiva individual. La sensibilidad puede aumentarse empleando reglas de predicción de la masa ósea; entre ellas, las más utilizadas son el osteoporosis risk assesment instrument (ORAI) y la simple calculated osteoporosis risk estimation (SCORE).

Existen otros métodos con la misma finalidad diagnóstica que la DMO, entre los que destacarían la densitometría por ultrasonidos y la tomografía computarizada cuantitativa. La densitometría por ultrasonidos presenta algunas ventajas (menor costo, rapidez, ausencia de radiación, etc.), pero los estudios publicados presentan datos de correlación, exactitud y precisión respecto a la DMO que limitan su utilidad. La tomografía computarizada cuantitativa presenta buenos resultados de validez, pero tiene unas limitaciones importantes (elevado costo, tiempo de realización, alta radiación, necesidad de personal especializado). ${ }^{(8)}$

Los niveles de Densidad Mineral Ósea según la OMS son los siguientes: (Ver tabla No. 1.)
Tabla No. 1: Clasificación de los niveles de Densidad Mineral Ósea.

\begin{tabular}{|l|}
\hline Normal: hasta -1 \\
\hline Osteopenia: entre -1 hasta -2.5 \\
\hline Osteoporosis: $>-2.5$ \\
Fuente: Organización Mundial de la Salud $(\mathrm{OMS})^{(7)}$
\end{tabular}

Se definió osteopenia como la densidad mineral ósea menor a la normal, con un T-score entre -1.0 y -2.5 y a la osteoporosis como la densidad mineral ósea con un T-score >- 2.5, según la OMS.

El objetivo del presente estudio fue la identificación de densidad ósea y riesgo de fracturas en pacientes que acudieron a Mediscan en el periodo de julio 2012 hasta enero 2016 para la realización de su densitometría ósea.

\section{PACIENTES Y METODOS}

Estudio descriptivo, retrospectivo, realizado en pacientes que acudieron a Mediscan en el periodo de julio 2012 hasta enero 2016 a estudio de Densitometría ósea.

Se atendieron un total de 2337 pacientes, de los cuales se excluyeron 20 por razones de interpretación fuera del Formato de Estudio o por datos incompletos, obteniendo un total de 2317 pacientes. Las variables que se obtuvieron para la realización del estudio fueron: edad, género, peso en kilogramos y talla. Cada paciente lleno un formulario de consentimiento informado permitiendo el uso de los datos obtenidos a través del examen de densitometría ósea para fines de investigación.

Para la realización del examen se utilizó una maquina: Discovery QDR Series. En este examen de Densitometría Ósea, se midió la densidad ósea en el cuello femoral y la columna lumbar, el paciente en decúbito supino en una mesa. El generador de rayos $X$ se encuentra ubicado debajo del paciente y un detector laser, posicionado arriba del paciente en el sitio marcado para rastro desde $L 1$ hasta $L 4$, en caso de la región de la columna lumbar 
y en la región intertrocantérica en el caso del cuello femoral, una vez obtenida las imágenes se transmiten a una computadora la cual hace la medición cuantitativa de las imágenes registradas posteriormente son enviadas al médico radiólogo para su interpretación.

El programa estadístico que se utilizó para la recolección de la muestra fue IBM SPSS Stadistic Program 22, realizándose un análisis estadístico tipo descriptivo de frecuencias y porcentajes.

\section{RESULTADOS}

Del total de pacientes, obtuvieron la siguiente distribución por género: femenino 2205 (95\%), masculino 112 (5\%). En el estudio se obtuvo la siguiente distribución por edades. (Ver tabla No. 2.)

Tabla No. 2: Distribución por edades.

\begin{tabular}{|l|l|l|}
\hline \multirow{2}{*}{ Edad } & \multicolumn{1}{|c|}{ Femenino } & Masculino \\
\cline { 2 - 3 } & No (\%) & No $(\%)$ \\
\hline 20-30 años & $17(0.7 \%)$ & $3(0.1 \%)$ \\
\hline $31-50$ años & $464(20 \%)$ & $21(0.9 \%)$ \\
\hline $51-60$ años & $694(29.9 \%)$ & $17(0.7 \%)$ \\
\hline $61-70$ años & $595(25.7 \%)$ & $40(1.7 \%)$ \\
\hline Más de 71 años & $435(18.7 \%)$ & $31(1.6 \%)$ \\
\hline Total & $2205(95 \%)$ & $112(5 \%)$ \\
\hline
\end{tabular}

Fuente: Instrumento Densitometría Ósea

Se encontró afectación ósea en las mujeres, en un $1530(66 \%) ; 54 \%$ presento osteoporosis y $12 \%$ osteopenia. Entre las que tenían más de 50 años; $11.82 \%$ presento osteopenia y $44.74 \%$ presento osteoporosis. (Ver tabla No. 3.)

Tabla No. 3: Género femenino y su Densidad Ósea.

\begin{tabular}{|l|l|l|l|}
\hline \multirow{2}{*}{ Edad } & \multicolumn{3}{|c|}{ Femenino } \\
\cline { 2 - 4 } & \multicolumn{1}{|c|}{ Normal } & Osteopenia & Osteoporosis \\
\hline $20-30$ años & $12(0.51 \%)$ & $1(0.04 \%)$ & $7(0.30 \%)$ \\
\hline $31-50$ años & $250(10.78 \%)$ & $7(0.30 \%)$ & $207(8.93 \%)$ \\
\hline $51-60$ años & $210(9.06 \%)$ & $69(2.97 \%)$ & $415(17.91 \%)$ \\
\hline $61-70$ años & $125(5.39 \%)$ & $97(4.18 \%)$ & $373(16.09 \%)$ \\
\hline Más 71 años & $78(3.36 \%)$ & $108(4.66 \%)$ & $246(10.74 \%)$ \\
\hline Total & $675(34 \%)$ & $282(12 \%)$ & $1248(54 \%)$ \\
\hline
\end{tabular}

Fuente: Instrumento Densitometría Ósea
De los hombres mayores de 50 años; $0.51 \%$ presento osteopenia y $1.97 \%$ osteoporosis.

(Ver tabla No. 4.)

Tabla No. 4: Género masculino y su densidad Ósea.

\begin{tabular}{|l|l|l|l|}
\hline \multirow{2}{*}{ Edad } & \multicolumn{3}{|c|}{ Masculino } \\
\cline { 2 - 4 } \multicolumn{1}{|c|}{ Normal } & Osteopenia & Osteoporosis \\
\hline 20-30 años & $1(0.04 \%)$ & $1(0.04 \%)$ & $2(0.08 \%)$ \\
\hline 31-50 años & $13(0.56 \%)$ & $2(0.08 \%)$ & $6(0.25 \%)$ \\
\hline 51-60 años & $7(0.30 \%)$ & $1(0.04 \%)$ & $9(0.38 \%)$ \\
\hline 61-70 años & $9(0.38 \%)$ & $5(0.21 \%)$ & $26(1.12 \%)$ \\
\hline Más 71 años & $10(0.43 \%)$ & $6(0.25 \%)$ & $11(0.47 \%)$ \\
\hline Total & $40(1.7 \%)$ & $15(0.6 \%)$ & $54(2 \%)$ \\
\hline
\end{tabular}

Fuente: Instrumento Densitometría Ósea

De la población general estudiada, $68.06 \%$ presentó baja densidad ósea; 297 (12.8\%) presento osteopenia y 1302 (56\%) presentó osteoporosis.

\section{DISCUSION}

La osteoporosis es una entidad clínica relacionada directamente con la edad. Su prevalencia aumenta con el envejecimiento y se duplica el riesgo de fractura por cada 10 años de edad. De esta forma, se estima que para una mujer de 50 años, el riesgo de padecer una fractura es cercano a $40 \% .^{(8)}$ La DMO es un Método diagnostico basado en una fuente de radiación con un haz de Rayos X focalizado, que estudia la columna lumbar y el cuello femoral, sitios anatómicos estándar para la evaluación de la densidad mineral ósea y que está en relación con una metodología numérica, que analiza $\mathrm{g} / \mathrm{cm} 3$ de hueso, usada para establecer el diagnostico de osteoporosis y predecir el riesgo de fractura. ${ }^{(6)}$

Pasados los 35 años, se alcanza el nivel máximo de calcio en el esqueleto humano, lo que se conoce como pico de masa ósea. A partir de este momento sigue una etapa en la cual la masa ósea ni aumenta ni disminuye. Con la llegada de la menopausia en la mujer, se inicia la etapa de pérdida de masa ósea que puede ser, en parte, amortiguada por el uso 
de terapia hormonal de reemplazo. A continuación le sigue un proceso de desmineralización que es menos marcado, pero que continúa inevitablemente hasta la muerte. ${ }^{\left({ }^{8}\right)}$

En el estudio se demostró que la mayor frecuencia de afectación ósea fue en el género femenino en un $66 \%$, esto apoyado por el marco teórico donde enuncia que uno de los factores que intervienen para la aparición de osteoporosis es el género femenino según la $\mathrm{OMS}^{(7)}$ en un $30 \%$, en un $15 \%$ mujeres con más de 70 años.

Aproximadamente $12 \%$ de las mujeres mayores de 50 años tuvieron Osteopenia, etapa intermedia antes de Osteoporosis, en la cual se pueden modificar factores nutricionales, dieta y ejercicio. Todos los pacientes con diagnóstico de Osteoporosis tenían riesgo de fractura en más de una desviación estándar, y los grupos más importantes fueron en las edades entre 60-70 años en ambos géneros y comparándolo con otros estudios en que la osteoporosis, se asocia con el envejecimiento y es predominante en la edad de 60 a 70 años. ${ }^{(9,10)}$

El $68.06 \%$ de la población estudiada presento baja densidad ósea, dentro de la cual, el $12.8 \%$ presento osteopenia y $56 \%$ presento osteoporosis lo que indica mayor riesgo de presentar algún tipo de fractura. Según la literatura el riesgo de fractura aumenta entre 1,5 y 3 veces o más por cada disminución de una desviación estándar (DE) de la DMO.
Comparándolo con otros estudios, se calcula que 10 millones de estadounidenses mayores de 50 años tienen osteoporosis, y que otros 34 millones están en riesgo de la enfermedad. Se estima que hay 1,5 millones de fracturas por fragilidad cada año. Si bien la mayoría de las mujeres menores de 50 años tienen una DMO normal, a la edad de 70 años el $27 \%$ tienen osteopenia y el $70 \%$ tienen osteoporosis en el cuello femoral, columna lumbar o en antebrazo. Existen diferencias étnicas y de género en la densidad ósea y en la incidencia de fracturas osteoporóticas; las mujeres tienen una densidad ósea menor que los hombres de la misma raza y después de la menopausia, la incidencia de fracturas es dos a tres veces mayor que en los hombres. $(10,11)$

En conclusión, existe una nueva preocupación por la Calidad de Vida y este debe de estar unida a la dieta, ejercicio y por eso conocer los factores de riesgo de nuestra población y segmentarla nos ayuda desde el punto de vista médico a ofrecer un mejor diagnóstico y una adecuada orientación a nuestros pacientes.

Se recomienda a los médicos promover la utilización del método de Densitometría Ósea como prueba para determinar tempranamente la densidad mineral ósea de los pacientes y valorar en forma oportuna el riesgo de fractura, para prevenirlas. 


\section{REFERENCIAS BIBLIOGRÁFICAS}

1. Sanfelix-Genovés J, Sanfelix-Gimero G, Peiró S, Hurtado I, Fluixà C, Fuertes A, et al. Prevalence of osteoporotic fracture risk factors and antiosteoporotic treatment and the Valencia region, Espain. The baceline characteristic of the ESOSVAL cohort.Osteoporos int. [Internet]. 2013. [citado el 3 de julio del 2016]; 24(3): 1045-55.

Disponible en: http://link.sprin ger.com/ar ticle/10.1007\%2Fs00198-01 2-2018-6.

2. Couto Núñez D, Napoles Mendez D, Deolofeu Betancout I. Osteoporosis posmenopausia según densitometría ósea. MEDISAN.[internet] 2011 [citado el 4 de abril del 2016];15(12):1765-74.

Disponible en: http://bvs.sld.cu/revistas/ san/vol_15_12_11/san121211.pdf.

3. Guía para el diagnóstico y tratamiento de la osteoporosis. Rev Cubana Endocrinol. [Internet] 2014 [citado el 18 de marzo del 2016];25(1):1-34. Disponible en: https:// www.iofbonehealth.org/sites/default/files/ pocket-guidelines-esceo-spani sh.pdf.

4. Naranjo A, Rosas J, Ojeda S, Salas E. Manejo de la osteoporosis en atención primaria antes y después del resultado de la densitometría osea; tratamiento instaurado versus tratamiento recomendado en los consensos (estudio CANAL). Reumatol Clin.[Internet]. 2013. [citado el 10 de enero del 2016];9(5): 269-273. Disponible en: http://www.reumatologiaclinica.org/es/ manejo-osteoporosis-atencion-prima ria-antes/articulo/S1699258X13000636/.

5. Sosa Hernández M, Gómez Díaz J. La osteoporosis, definicion, importancia. fisiopatologia y clinica. Rev Osteoporosis Metab Miner. [Internet]. 2010 [citado el 20 de diciemre del 2015];2(sple 5): S3-S7. Disponible en: http://www.revistadeosteo porosisymetabolismomineral.com/pdf/ articulos/92010020500030007.pdf.
6. Salazar Madrigal K. Osteoporosis un problema mayor de Salud Publica; Rev. costarric. salud publica.[Internet]. 2008. [citado el 4 de marzo del 2016];17(32): 75-79. Disponible en: http://www.scielo. sa.cr/scielo.php?script=sci_arttext\&pid= S1409-14292008000100010.

7. Hermoso de Mendoza MT. Clasificacion de osteoporosis, factores de riesgo, clinica y diagnostico diferencial. Anales. Sis San Navarra. [internet]. 2003. [citado el 20 de marzo del 2016];3(1): 29-52.

Disponible en: http://scielo.isciii.es/pdf/ asisna/v26s3/original3.pdf.

8.

Orueta R, Gómez-Caro S. Interpretación de densitometría ósea. Semergen. [Internet]. 2010. [citado el 17 de enero del 2016]; 36(1):27-30. Disponible en: http://www. elsevier.es/es-revista-semergen-medici na-familia-40-articulo-interpretacion-densi tometria-osea-S1138359309000069.

9.

Carranza-Lira S, Lanuza-López MC, Sandoval-Barragán MP. Comparacion del riesgo de fractura calculado con la herramienta FRAX con y sin densitometria osea en un grupo de mujeres mexicanas. Ginecol Obstet Mex.[Internet]. 2014. [citado el 3 de abril del 2016]; 82(1): 591-594. Disponible en: http://www.medigraphic.com/pdfs/ ginobsmex/gom -2014/gom149c.pdf.

10.

Molina Restrepo JF, González Naranjo LA. Osteoporosis: enfoque clínico y de laboratorio. Medicina y Laboratorio. [Internet]. 2010. [citado el 7 de marzo del 2016];16(3):111-140. Disponible en: http:// www.medigraphic.com/pdfs/medlab/ myl-2010/myl103-4b.pdf.

11.

González LA, Vásquez GM, Molina JF. Epidemiologia de la Osteoporosis. Rev Colomb. Reumatol. [Internet]. 2009. [citado el 20 de agosto del 2016]:6(1):61-75. Disponible en: file:///C:/Users/Invitado/Downloads/ S0121812309701197_S300_es\%20(1).pdf. 